\title{
Abordajes interdisciplinarios de mujeres con mujeres: escenas de la vida carcelaria
}

Interdisciplinary approaches of women with women: scenes of prison life

Serrana Mesa Varela

Universidad de la República. Facultad de Humanidades y Ciencias de la Educación.

Centro de Estudios Interdisciplinarios Uruguayos, Uruguay

serranamesa@gmail.com

Natalia Montealegre Alegría

Universidad de la República. Facultad de Humanidades y Ciencias de la Educación.

Centro de Estudios Interdisciplinarios Uruguayos / Area de Derechos Humanos - Servicio Central de

Extensión y Actividades en el medio (SCEAM), Uruguay

montealegre.alegria@gmail.com

Flor de María Meza Tananta

Universidad de la República. Instituto de Derechos Humanos de la Facultad de Derecho y Área de Derechos

Humanos del Servicio Central de Extensión y Actividades en el medio (SCEAM), Uruguay

flormameza@gmail.com

\section{ReSUMEN:}

Este artículo presenta el desarrollo de una experiencia interdisciplinaria de un equipo de investigadoras de la Universidad de la República (Uruguay) con foco en los derechos humanos de las mujeres prisioneras en el país. La reflexión se sustenta en dos ámbitos de trabajo: un curso con operadores y policías penitenciarios y un taller de "formación de formadoras" con mujeres recluidas en la Unidad № 5. A lo largo del texto se problematizan de qué modo el giro punitivo en el país, el concepto de mujer y la relación con la alteridad se encuentran con un abordaje académico feminista.

Palabras ClaVe: Cárcel, Mujeres, Interdisciplinariedad, Feminismo, Derechos Humanos, Uruguay.

\section{Abstract:}

This article presents the development of an interdisciplinary experience by a team of female researchers from the Universidad de la República, with a focus on the human rights of imprisoned women in Uruguay. The deliberation is based on two areas of work: a course with police force and penitentiary staff and a "formación de formadoras" (training of trainers) workshop with women who are imprisoned in Unit number 5. Throughout the text, we problematize how the punitive turn in the country, the concept of women and the relationship with otherness meet with a feminist academic approach.

KEYWORDS: Prisons, Women, Interdisciplinarity, Feminism, Human Rights, Uruguay.

\section{INTRODUCCIÓN}

En este artículo presentamos el desarrollo de una experiencia integral e interdisciplinaria de un equipo de investigadoras de la Universidad de la República con foco en los derechos de las mujeresprivadas de su libertad ambulatoria por conflictos con la ley, ${ }^{1}$ presas en la Unidad Penitenciaria $N^{\circ} 5$ de la región metropolitana 
del departamento de Montevideo, Uruguay. Estas prácticas nos han llevado a problematizar -junto con los marcos y las metodologías específicas - los sentidos en torno al concepto de mujer, la relación con la alteridad (y la mismidad) y de qué modo ambos elementos se encuentran con un abordaje situado de académicas feministas con posicionamientos diversos.

Constituye un desafío pensar la prisión focalizando en las relaciones de poder atendiendo a la centralidad que el género cobra en cómo y qué relaciones sociales se imbrican en la interna del Sistema Penitenciario. E implica que cuando hablemos de la cárcel desde un punto de vista teórico, no recurramos a nociones androcéntricas desde las que se analizan las cárceles tradicionalmente. A esa complejidad, se suma que las autoras del texto reflexionaremos en torno a una experiencia de investigación, enseñanza y extensión universitaria desarrollada durante los últimos años, ${ }^{2}$ acompañando el proceso de "cambio institucional" ${ }^{3} \mathrm{y}$ la puesta en práctica de una política carcelaria que es enunciada como "humanizadora del sistema", pero que en su implementación está plagada de tensiones y dificultades. Se trata de un contexto en el que la categoría "mujer", con sus diversos pliegues y espesores, se revela con todas sus complejidades.

Si bien es sabido que cuando nos referimos a "mujeres" nos encontramos trabajando con una categoría no monolítica ni heterogénea -y que dentro de las complejidades para su abordaje será pertinente considerar la pertenencia étnico racial, de clase, religiosa, de migrante, entre otros aspectos socio culturales - en la situación misma de incorporación al dispositivo carcelario, algunos de sus límites se tensionan expresando de un modo más claro la labilidad de esa condición y sus características singulares.

Problema complejo por el tipo de dislocación que produce, en particular en el encuentro -el diálogo intersubjetivo- cuando se propone como epicentro el universalismo de los derechos humanos, como veremos en las instancias "en terreno" - dos dispositivos de taller desde una perspectiva de derechos- que se analizarán a lo largo del artículo.

Para comprender este proceso de trabajo, es menester, en primer lugar, desarrollar algunos elementos vinculados a la realidad carcelaria en Uruguay a la que refiere la reflexión que aquí presentamos.

\section{Del DisPositivo CARCELARIO}

En Uruguay, como en otros países, la cárcel es una institución de castigo, un espacio de sanción social, el lugar en el que se penan las transgresiones a las normas con la privación de la libertad, sin que el Estado garantice ${ }^{4}$ plenamente los derechos humanos de las personas bajo su custodia. ${ }^{5}$ El crecimiento constante del índice de personas privadas de libertad por habitante ha aumentado junto a la multiplicación del interés por el tema. ${ }^{6}$ Sin embargo, este crecimiento no se da de la misma forma entre varones y mujeres, aumentando, durante la primera década del siglo XXI, en forma exponencial, el porcentaje de mujeres en comparación con el de los varones. En 1998, había veintidós varones privados de libertad por cada mujer, en 2010, eran 11 los recluidos por cada mujer presa (Vigna, 2012, p. 18). En el nivel nacional, este proceso ha tenido lugar de modo sostenido hasta noviembre de 2017, cuando se inicia la implementación de un nuevo Código del Proceso Penal (CPP). Este debía incidir paulatinamente en la reducción del número de personas privadas de libertad, en particular, por el llamado uso indiscriminado y constante de la prisión preventiva, vale decir, el uso rutinario de la prisión como medida a ser aplicada desde la detención hasta la condena. Uruguay ha recibido observaciones de organismos internacionales por el uso abusivo de la prisión preventiva. ${ }^{7}$ En 2011 , la Comisión Interamericana de Derechos Humanos (en adelante, CIDH) le "instó a promover un cambio en la cultura y la práctica judicial establecidas, que produzca un verdadero cambio de paradigma en la concepción de la procedencia y necesidad de la prisión preventiva" (CIDH, 2017, p. 22).

El nuevo CPP intentó limitar esa rutinización del encarcelamiento en pos de la implementación de otro tipo de medidas alternativas, atendiendo principalmente que este "Código reconoce expresamente el derecho a ser tratados con el respeto debido a la dignidad de ser humano a todos los que intervienen en el proceso, en 
especial a la víctima del delito y a la persona a quien se le atribuye haberlo cometido" (Dirección Nacional de Impresiones y Publicaciones Oficiales, en adelante IMPO (2018, s/p).

Ese código, en el correr de 2018 y a instancias del Poder Ejecutivo, fue modificado limitando las opciones de los jueces para la no prisionización de las personas (Larrobla y Mesa Varela, 2018), en particular en el caso de reincidencia en delitos contra la propiedad.

Por otra parte, en lo que respecta a las personas privadas de su libertad por conflictos con la ley, tradicionalmente se ha pensado y se han diseñado políticas dirigidas a ellas en clave masculina, tal como lo ha planteado de manera pionera Antony (2000). Esto, porque la representación generalizada de la persona privada de libertad es "un preso": hombre, pobre, con determinado babitus (Bourdieu, 1967, p. 152) que condensa el estigma (Goffman, 2009) y el prestigio de cierta masculinidad subalternizada; dejando fuera de aquello a considerar tanto a mujeres como a trans, como a personas pertenecientes a otros sectores sociales o portadoras de otros capitales materiales y simbólicos (Bourdieu, 1987).

Según algunos argumentos (Almeda y Bodelón, 2007; Antony, 2000; entre otrxs), el androcentrismo en el tratamiento de las personas privadas de libertad (tanto desde lo político, como desde lo académico), se ha sustentado en el bajo número de mujeres encarceladas. El gran aumento que se observa en los últimos años, ha llevado a que este sector de la población reclusa haya dejado de ser completamente invisible (Antony, 2007). Esto ha mostrado la necesidad de que se piense en políticas específicas y, también, ha llevado a conocer sus condiciones particulares (Almeda y Di Nella, 2017), encontrándonos en lo últimos años frente a variaciones en la política criminal hacia las mujeres.

Este proceso hace necesario entender sus causas, las consecuencias en la vida social, las posibles consecuencias de las variaciones en las políticas carcelarias implementadas y a implementar. Especialmente, en el marco de la reforma uruguaya establecida por la creación del Instituto Nacional de Rehabilitación (INR), a través de la ley No 18.719 , aprobada en diciembre de 2010. Esta ley establece la supresión de la Dirección Nacional de Cárceles y la unificación del sistema penitenciario a través de la creación del mencionado Instituto. "Su cometido principal es operar de institución rectora de la política penitenciaria nacional, bajo tres ejes de actuación definidos: seguridad, tratamiento y gestión” (Uruguay. Ministerio del Interior, 2011, p. 11). Como parte de este proceso, en el año 2011 se comienza con un plan de retiro de las cárceles de la administración policial, buscando independizar al sistema penitenciario del Ministerio del Interior. ${ }^{8}$

Es así que, desde la academia en Uruguay, las cárceles para mujeres -dejando de lado los estudios sobre los establecimientos militares de reclusión para prisioneras políticas (1968-1985) - han sido estudiadas tardíamente, particularmente a partir de fines de la década de los '90 del siglo pasado. Estos trabajos se han concentrado en las instituciones penitenciarias de mujeres como unidad de análisis. Han tomado como objeto de estudio su población, focalizando en cuestiones como: visibilizar las particularidades de la privación de libertad en las mujeres, la preeminencia de los estereotipos de género en el tratamiento carcelario (fundamentalmente en lo que se vincula al trabajo y a la educación), el plus de la pena, el destino y futuro de los hijos e hijas de las mujeres privadas de libertad, entre otros (Antony, 2000, 2007; Scarfo, 2003). Estos enfoques resultaron imprescindibles como punto de partida, ya que existía un casi total desconocimiento de estas instituciones y su población femenina.

Como ejemplo, en 2005, se crea la Mesa de Trabajo sobre Mujeres Privadas de libertad ${ }^{9}$ (CLADEM, 2008; Folle y Mesa Varela, 2016) para conocer, precisamente, la situación de esa parte de la población en Uruguay: número, ubicación, condiciones de prisionización, convivencia con niños y niñas, entre otros aspectos que hoy son de público conocimiento en el país.

Un informe realizado ese año por esa Mesa expone, por primera vez, las características de las mujeres privadas de libertad en Uruguay (Mesa de Trabajo sobre Mujeres Privadas de Libertad, 2005). Más adelante, aparecen investigaciones más específicas, que incluyen temas como análisis de las causas por las que las mujeres han crecido dentro de la población reclusa total, la generización del delito, su participación en las visitas, entre 
otros temas (Folle, 2011; Folle y Mosquera, 2016; Mesa Varela, 2012, 2016, 2019; Montealegre Alegría, 2016b; Vigna, 2008, 2011a y 2011b).

Este recorrido por la producción de conocimiento desde la academia y la sociedad civil sobre la temática, hizo visible, en primer lugar, la existencia de mujeres privadas de libertad, intentando romper con el "mito" igualador preso-hombre, para después mostrar sus particularidades y necesidades específicas.

Sin embargo, en el Uruguay contemporáneo -y en consonancia con la situación política en la región - nos encontramos en un contexto que nos interpela profundamente desde dos ángulos: la punitividad creciente en el país ${ }^{10}$ y las luchas de las mujeres y los feminismos para lograr cambios profundos y duraderos en las relaciones de poder entre los géneros y en la sociedad toda, incluyendo sus instituciones; procesos, ambos, de creciente interés.

Respecto a la punitividad -como uno de los dos nodos centrales que tensionan el análisis de la situación de las mujeres presas en Uruguay- vale precisar que lo entendemos con la complejidad desarrollada por Wacquant en Castigar a los pobres, haciendo énfasis en los aspectos simbólicos del castigo (Wacquant, 2010). Aspectos que también hacen posible cohesionar a sectores de la población, cercando al mismo tiempo que creando lealtades de diverso orden. Proceso en el que "las élites políticas refuerzan e incluso anticipan las inquietudes de seguridad de los ciudadanos. (...) Ayudados en eso por el tratamiento mediático de las noticias y de los acontecimientos violentos, esas élites acompañan, exacerbando, incluso suscitando las ansiedades y los miedos. Los instrumentalizan" (Fassin, 2018, p. 17).

En Uruguay, estas prácticas, en el presente, encuentran su correlato en la campaña "Vivir sin miedo" que ha obtenido el número de firmas suficientes para la consideración por voto directo. La iniciativa fue lanzada públicamente en mayo de 2018 por el senador del Partido Nacional ${ }^{11}$ Jorge Larrañaga (Alianza Nacional), con el objetivo de modificar algunos artículos de la Constitución de la República Oriental del Uruguay para reforzar la seguridad. Para ser plebiscitada, necesitaba llegar a 10\% del padrón electoral (270.000 firmas de ciudadanos uruguayos en condiciones de votar) y fue presentada, finalmente, con 376.427 adhesiones.

Estos nudos centrales de la coyuntura se entrelazan con el abordaje del trabajo realizado con y para las mujeres privadas de libertad. A pesar de que estos dos hechos son fácilmente comprobables -el aumento del castigo $^{12}$ y de la demanda social del mismo, y el crecimiento de las luchas de las mujeres en el Uruguay ${ }^{13}$ - los significados atribuidos a ellos son variables y resulta particularmente interesante la confrontación de nuestros sentidos - de quienes desde este equipo de trabajo ingresamos a trabajar con mujeres privadas de libertad sobre sus derechos como tales- con los de las mujeres presas con las que realizamos talleres y encuentros.

Pero la unión de estas crecientes luchas con los intereses de las mujeres privadas de libertad, no se ha dado tan clara ni directamente. Sigue, por tanto, resultando necesario visibilizar la situación de las mujeres presas, no como "sobre explotación victimista", sino como "práctica de resistencia" (Guzmán y Platero, 2012, p. 127), fundamentalmente desde quienes elaboramos este trabajo. En consonancia con lo planteado por Almeda y Di Nella (2017, p. 184) respecto a que cada "vez más, muchas de las investigadoras se implican en instituciones gubernamentales o en las realidades de las presas, construyendo, conjuntamente con ellas, resistencias y alternativas a la opresión y a la exclusión que, hoy por hoy, representa la cárcel, el control y el castigo a las mujeres".

Seguimos resistiendo -desde el trabajo académico - a que las mujeres no sean tenidas en cuenta en las políticas criminales y penitenciarias, a que las personas que trabajan con ellas tengan encarnada irreflexivamente la desigualdad de poder basada en clase y género, a que como académicas feministas no comprendamos profundamente cómo la lucha por los derechos de las mujeres es vivida y significada por aquellas que están privadas de la libertad por conflictos con la ley.

Creemos entonces que necesitamos ir un paso más allá. Ya no alcanza con visibilizar -y combatir - una situación desventajosa en lo que atañe a las especificidades de las mujeres privadas de libertad, sino analizar en profundidad qué estamos dejando fuera de la complejidad de identidades, representaciones y relaciones que 
aparecen en la población recluida en la cárcel para mujeres, al trabajar con ellas y visibilizar su situación desde la producción académica.

Asimismo, aparece como necesario pensar cómo impacta esta "época del castigo" o "giro represivo" (Fassin, 2018 , p. 9) que se ha profundizado en el Uruguay de los últimos años -con una gran maquinaria mediática y política sosteniéndola - en las identidades y en los posicionamientos de las mujeres privadas de libertad. Pero se trata también de eludir la fijación en una identidad estigmatizante, que se encarna de forma muy potente en esos cuerpos y subjetividades; sin desconocerla ni eliminarla, intentando analizar qué rol juegan las relaciones de poder y de género allí.

Por estos motivos, atendiendo a la sociología del campo (Bourdieu y Wacquant, 1992), se estableció trabajar en distintas instancias con interlocutores e interlocutoras que se identifican como claves: autoridades del sistema (Departamento de Género y Diversidad del INR, dirección de la Unidad penitenciaria No 5 y Centro de Formación Penitenciaria -en adelante, Cefopen-), operadores penitenciarios, policías a cargo de las revisorías y mujeres recluidas en la unidad No 5 . A los efectos de este texto, seleccionamos dos experiencias que resultan pertinentes para la reflexión propuesta y que presentaremos sintéticamente a continuación. Se trata del curso "Derechos humanos en práctica" dirigido a policías y operadores penitenciarios y el taller con mujeres presas en torno a sus derechos como personas, como mujeres, como privadas de libertad.

\section{Derechos humanos en práctica}

La experiencia de trabajo con operadores, operadoras y policías adscritos a cárceles que aquí compartimos, contó con un proceso de gestión y negociación junto a la dirección del Cefopen que es, en sí mismo, un insumo para la investigación del equipo. Además de las problemáticas centrales detectadas por la Dirección de Cefopen, a partir de las reuniones realizadas, fue posible aproximarse a algunas de las tensiones en torno a la formación de operadores penitenciarios, las relaciones de poder entre policías, operadores y operadoras -signadas por las relaciones de género y vertebradas por la dominación masculina, que se vuelven profundamente explícitas al ingresar a trabajar en los dispositivos carcelarios (Tait, 2011) - y la identificación de los puntos críticos de vulneración de derechos ya identificados por la institución.

Sin ánimo de detenernos en este aspecto, interesa precisar que la solicitud de ajuste de la propuesta inicial por parte de la dirección tuvo dos argumentos centrales: en primer lugar, la falta de un sistema de educación permanente para personal adscrito a cárceles (policías y operadores independientemente de la capacitación previa o no de cada uno y de las características de los contenidos y tiempos formativos de cada generación); y, segundo, la necesidad de reducir los niveles de violencia desplegados en revisorías por la centralidad que ese ámbito adopta en la relación entre las mujeres privadas de su libertad y sus relaciones interpersonales con el "afuera" de la cárcel. El abordaje del complejo entramado que constituyen las relaciones sociales en las que se desarrolla la privación de la libertad de las mujeres en Uruguay - mucho menos visitadas que los varones presos- ${ }^{14}$ con raíz en su transgresión o conflicto con la ley, no puede dejar de considerar a las visitas de sus allegados y los ritos de paso por los que deben transitar (Montealegre Alegría, 2016a).

El dispositivo propuesto con las características antes señaladas se sustentó: en los resultados de investigaciones anteriores, en las instancias de negociación con las autoridades como fuente de información sobre la dinámica interna y en los perfiles funcionariales de los participantes. Sobre esa base, adecuamos las estrategias didácticas en el abordaje de los derechos humanos partiendo del desarrollo histórico de las políticas punitivas, habilitando la incorporación de los tópicos emergentes en cada encuentro a través de la construcción de un "espacio seguro" que hizo posible la conformación de un grupo. Esto es un elemento indispensable para cumplir con los objetivos de la instancia práctica desarrollada en el ámbito carcelario propiamente dicho como veremos a continuación. 


\section{LA DIGNIDAD EN CUESTIÓN}

Para abordar la estrategia teórico-metodológica desplegada, es necesario explicitar algunos de los contenidos normativos que integraron el corpus conceptual del curso. Partimos de una constatación, que podría resultar obvia: quienes están privadxs de libertad y quienes los visitan son personas y en tanto tales tienen derechos y libertades fundamentales reconocidos por el ordenamiento jurídico uruguayo, el sistema interamericano y el sistema universal de los derechos humanos. Estos ordenamientos jurídicos deben asegurar, promover y garantizar "las exigencias de libertad, la igualdad y la seguridad humanas en cuanto expresión de la dignidad del ser humano" (Nogueira, 2003, p. 4).

De tal modo que el único derecho que teóricamente se limita a las personas privadas de libertad jurídicamente es el de su libertad ambulatoria. Todos los demás derechos siguen vigentes, especialmente a "ser tratadas humanamente, y a que se respete su dignidad, su vida, su integridad física, psicológica y moral” (CIDH, 2009, p. 1).

Estos derechos humanos de las personas privadas de libertad -haciendo especial énfasis en el derecho a la dignidad, los instrumentos internacionales del derecho internacional de los derechos humanos, las reglas mínimas existentes en ambos sistemas sobre los derechos de las personas privadas de libertad y las obligaciones del Estado- fueron trabajados en la teoría y confrontadas con la práctica en la visita a la unidad $\mathrm{N}^{\circ} 5$ que hicimos, docentes del taller arriba mencionado y lxs participantes del Curso "Derechos humanos en práctica".

Con ese marco también trabajamos el concepto de dignidad y el contenido del Protocolo de Inspecciones del INR. Instrumento que aún se encuentra en etapa de revisión pero que ya fue distribuido a las autoridades de las unidades penitenciarias para su puesta en práctica.

Hacer inteligible la teoría, el "deber ser" de la norma, en un espacio de conflicto como es la cárcel es una tarea compleja pero no por ello imposible. El ejercicio concreto que realizamos, dialogando sobre el concepto de dignidad y "pasando" por el escáner nos obligó a "poner los pies en la tierra" para constatar que entre la norma pautada, es decir el derecho uruguayo vigente -que por supuesto incluye a todos los instrumentos de derechos humanos - y la práctica de la revisoría para quienes visitan a las personas privadas de libertad hay una enorme brecha. En consecuencia, se producen un conjunto de vulneraciones de derechos sobre cuerpos concretos, sexualizados y generizados, que podrían nominarse o conceptualizarse, también, como el no respeto de la dignidad de las personas.

¿Sobre qué concepto de dignidad se trabajó? El de aquella que estructura el concepto mismo de derechos humanos. Así lo define Pedro Nikken "Una de las características resaltantes del mundo contemporáneo es el reconocimiento de que todo ser humano, por el hecho de serlo es titular de derechos fundamentales que la sociedad no puede arrebatarle lícitamente" (Nikken, s/f). Esto se encuentra consagrado desde 1948, en el artículo 1 de la Declaración Universal de los Derechos Humanos cuando señala que todos los seres humanos nacen libres e iguales en dignidad y derechos.

Si seguimos esta doctrina, se entiende que la dignidad se constituye en la fuente de todos los derechos humanos, tal como afirma Nogueira (2003), y en consecuencia debe rechazarse el ejercicio de cualquier derecho que suponga un atentado a ella. En ese sentido la dignidad y los derechos humanos de todas las personas que habitan un territorio es consustancial a un Estado de derecho.

Al mismo tiempo ese Estado, ejecuta a través de un funcionariado "su mandato" y encarna aquellas normas y políticas no explicitadas en torno a cómo se considera o no a esos otros, limitando sus condiciones de posibilidad a la dicotomía hombre-mujer. En este sentido, el siguiente fragmento de diario de campo resulta esclarecedor respecto a las limitaciones de representación de los cuerpos y las personas en ese contexto de acción:

Cuando todo el grupo estaba ya en la zona previa a los escáneres, luego de haber entregado los documentos de identidad, a pedido del equipo docente, una funcionaria policial de revisoría tomó la palabra y apoyó el ejercicio de poner los derechos en práctica en base a la descripción de la labor que allí realizan. 
Explicó las razones de los criterios utilizados en esa Unidad para llevar a cabo las revisaciones corporales, las que se basaban fundamentalmente en la seguridad y en evitar el ingreso de sustancias u objetos prohibidos al recinto carcelario. También relató las dificultades que encontraban en el desarrollo de sus tareas ejemplificando con la situación de la visita de una mujer trans. Explicó que, en ese caso, intervenían dos funcionarios, una mujer y un varón. La parte superior del cuerpo era revisada por la funcionaria y la de abajo por el funcionario porque, según afirmó: <la compañera también tiene derechos>. Los senos propios del sexo femenino debían ser expuestos, levantados, frente a ella mientras que la genitalidad masculina debe ser enajenada de su vista (Diario de campo, 17 de julio de 2018).

¿Qué derechos, entonces, y para quiénes? Además de contar jurídicamente con todos los instrumentos de derechos humanos de ambos sistemas, regional y universal, y las normas nacionales, también se dispone del Protocolo de Inspecciones del INR que tiene referencias a la inspección de las visitas a lo largo de su articulado, especialmente en el artículo 20 donde se enfatiza que esta inspección se realizará manteniendo el respeto y la dignidad de las personas.

Es importante resaltar que, sobre los derechos de quienes visitan a las personas privadas de libertad existen Reglas en el ámbito de las Naciones Unidas, como las Reglas Mandela, y los Principios y Buenas Prácticas sobre la Protección de las Personas Privadas de Libertad en las Américas. En ambos instrumentos, nos detendremos para precisar los alcances de las mismas problematizando al cierre su puesta en práctica.

En las Reglas Mínimas de las Naciones Unidas para el Tratamiento de los Reclusos, -también denominadas, Reglas Nelson Mandela- respecto al contacto con el mundo exterior, Regla 58 y Regla 60, se explicita que "Los procedimientos de registro y entrada no podrán ser degradantes para los visitantes y se regirán por principios cuando menos tan protectores como los que figuran en las reglas 50 a 52 . Se evitarán los registros corporales y no se emplearán con niños" (United Nations Office on Drugs and Crime -en adelante, UNODC, 2015, p. 19). Las reglas 50 a 52 se refieren a los registros de reclusos y celdas señalando expresamente que "Los registros se realizarán de un modo que respete la dignidad intrínseca del ser humano y la intimidad de las personas, así como los principios de proporcionalidad, legalidad y necesidad" (UNODC, 2015, pp. 16-17).

Asimismo, se consigna que los registros no se utilizarán para intimidar ni inmiscuirse en la intimidad; que los registros invasivos, como los registros personales sin ropa y los registros de orificios corporales, solamente se llevarán a cabo cuando sean absolutamente necesarios, que se harán en privado y por personal calificado del mismo sexo que el recluso. Se deja claro que este personal calificado no debe ser el mismo responsable de la atención del recluso, sino que debe ser personal médico calificado o, como mínimo, miembros del personal debidamente capacitado por profesionales médicxs respecto a normas de higiene, salud y seguridad (UNODC, 2015, pp. 48-49). Entonces, por una parte, el no cumplimiento de estas normas a través, por ejemplo, de la doble revisión descrita supone un tipo de vulneración. Por otra parte, la nomenclatura misma de estas reglas coadyuva al mantenimiento de un imaginario dicotómico anclado en el dimorfismo sexual, condenando a lo no clasificable a las personas trans.

Respecto al contacto con el mundo exterior, el principio número XVIII de los "Principios y buenas prácticas sobre la protección de las personas privadas de libertad en las Américas” establece el derecho de las personas privadas de libertad a "mantener contacto personal y directo, mediante visitas periódicas, con sus familiares, representantes legales, y con otras personas, especialmente con sus padres, hijos e hijas y con sus respectivas parejas" (CIDH, 2009). Agrega, en el principio XX, que el personal encargado de la custodia, tratamiento, y vigilancia, entre otras, de personas privadas de libertad debe ajustarse al respeto de los derechos humanos de las personas privadas de libertad y de sus familiares.

La vulneración de los derechos de familiares y personas que visitan, en especial el derecho a la integridad personal, ha merecido el reconocimiento y pedido de reparación de la Corte Interamericana de Derechos Humanos en numerosa jurisprudencia. ${ }^{15}$ Desde un posicionamiento en clave de derechos humanos, acortar la brecha entre la norma y la práctica es una meta consensuada.

$\mathrm{Al}$ mismo tiempo, en la práctica laboral a la interna de los recintos carcelarios -según los participantes del taller - respetar los derechos de las personas privadas de su libertad implica el riesgo de ser tildado por los 
otros como propichi. ${ }^{16}$ Todo este pasaje indigno de la revisoría podría evitarse con un body scanner, nos dicen. El espacio para la revisoría es pequeño. Allí se amontonan visitantes y la guardia o el guardia (o ambos, en el caso de una persona trans) que revisa, ordena semidesnudarse, observa, hace agachar y toser, sacar la lengua y moverla en cuclillas y con los brazos detrás del cuello, todos movimientos y posiciones sexualizados y de subordinación. Allí, frente a la autoridad, se evalúan los cuerpos que ingresan al dispositivo carcelario, dejando en evidencia sus adecuaciones o no a las normas y presentación de género (De Lauretis, 2000). La sucesión de espacios de revisación, cubículos de aproximadamente cuatro metros cuadrados, previos al ingreso al espacio carcelario propiamente dicho, funcionan también como "cabinas de vigilancia del género" (Preciado, 2009, p.14). Quienes participan de este proceso de taller, sienten que en la revisoría el Estado lxs castiga a todxs: presos, presas, visitas, guardias, operadores y operadoras penitenciarias.

\section{Cruzando alambrados: de los derechos a las mujeres}

El antecedente y fundamento principal para la elaboración del dispositivo de trabajo en talleres con mujeres privadas de libertad de la Unidad $N^{\circ}$, fue la Guía de derechos para mujeres privadas de libertad, cuyos contenidos fueron elaborados por Flor de María Meza (2016a; 2018) junto con aportes del resto del equipo. ${ }^{17}$ En esta guía, se recogieron y expusieron de forma amigable -no sólo para la población reclusa, sino también para las autoridades- ${ }^{18}$ los derechos específicos de las mujeres privadas de libertad.

Dado que el acceso y la apropiación de los contenidos de la guía por parte de la población recluida se fueron revelando como dificultosos, es que se pensó en generar instancias para que este material fuera difundido adecuadamente entre diversos participantes de la dinámica carcelaria, tanto para propiciar un mayor acceso de las mujeres al conocimiento de sus derechos, como para dinamizar y ajustar el trabajo de los operadores y operadoras penitenciarias en algunos aspectos. Para lograr concretar el trabajo de difusión y apropiación de la Guía en la unidad No 5, solicitamos reuniones y permisos con las diversas autoridades tanto de la Unidad como del INR. La encargada del área de género del recinto carcelario, una operadora penitenciaria, se convirtió así en nuestro enlace con la población reclusa y gestionó los horarios, las locaciones, las convocatorias, entre otros apoyos.

Para la elaboración del trabajo en talleres sobre la guía, se partió de la hipótesis -que interesa problematizar - que plantea que "la mayoría de las mujeres privadas de libertad, llegan a la institución sin conocimientos de sus derechos como personas, como mujeres y, mucho menos, como mujeres en situación de privación de libertad" y que "Conocer sus derechos se establece como una condición sine qua non para una permanencia con mayor dignidad en la cárcel” (Meza, 2016b).

Sobre esas afirmaciones, se consideró, además, fundamental que las propias mujeres privadas de libertad pudieran manejar y transmitir esta información, para hacerla llegar a sus pares. Es así que se elaboró una propuesta para la "formación de formadoras" que incluía 5 talleres (finalmente, se realizaron 6), de entre una hora y media y dos horas de duración, con frecuencia semanal. Se preveía que participaran entre 10 y 15 mujeres presas de diferentes sectores de la Unidad (desde máxima seguridad a máxima confianza) para que la replicación de las instancias de taller abarcase a la mayor parte de las reclusas.

Los contenidos previstos para desarrollar en los talleres fueron: cárcel, privación de libertad, definiciones y vivencias, género, privación de libertad desde una perspectiva de género, derechos de las mujeres y de las mujeres privadas de libertad, y replicación de contenidos.

Más allá de tener objetivos definidos para este proceso, se consideró importante utilizar una metodología de trabajo abierta a las propuestas y necesidades de las mujeres participantes como parte de la búsqueda de co-construcción de metodologías adecuadas a la realidad intracarcelaria. De tal forma que la planificación de los encuentros estaba abierta a una reconfiguración in situ a partir de lo que la situación pudiera demandar 
-para la emergencia espontánea de temas, propuestas e inquietudes - sin dejar de tener como horizonte los objetivos que nos habíamos propuesto.

Por ello, planificamos estructuras y encuadres amplios con una caja de herramientas que iba desde técnicas de relajación y yoga, técnicas de improvisación teatral a dinámicas grupales y didácticas. Como equipo consideramos que lo más importante para conseguir los niveles de participación y reflexión deseados era generar un buen vínculo entre todas las participantes (incluyéndonos) en el marco de un ambiente de confianza.

En base a este proceso, nos interesa reflexionar sobre dos situaciones que se dieron durante la realización de los talleres y que nos permiten hacer un ejercicio de análisis de las dinámicas de relacionamiento e identidad en el ámbito carcelario, así como un ejercicio de reflexividad ${ }^{19}$ sobre nuestra propia práctica. Fundamentalmente, son situaciones que nos interpelan a nosotras y nuestras (pre) nociones, que nos llevan a reflexionar sobre el lugar y el alcance de una práctica feminista en ese ámbito y sobre todo en la necesidad de un trabajo profundo de reconocimiento desde las diferencias existentes entre feminismos a la interna de nuestro propio equipo universitario. En el entendido de que "ser ético es acoger la interpelación del intruso, del diferente, en el nosotros de la comunidad moral, si el forastero, en su intervención, no puede o no podría tener control material sobre las condiciones de nuestras existencias, es decir, cuando no interviene en nuestra vida a partir de una posición de mayor poder." (Segato, 2013, pp. 11-12). Nuestra premisa era no imponer visiones ni posicionamientos, sino, a partir de un corpus establecido -el derecho internacional de derechos humanos y específicamente de las mujeres y de las mujeres privadas de libertad- discutir e intentar hacer emerger la necesidad de un trabajo específico y situado de reivindicación de derechos.

En varias ocasiones, durante el desarrollo de los talleres, incluso cuando ya llevábamos varios encuentros trabajando el tema, más de una de las participantes nos planteó no comprender por qué hablábamos de los derechos de las mujeres privadas de libertad y no de los derechos de las mujeres, y -principalmente- de los derechos de todas las personas privadas de libertad. Los planteos y argumentos dados, exponían que la identidad como mujeres era reconocida y había allí entonces la posibilidad de acuerdos sobre las demandas específicas y sus particularidades, diferentes a las de los hombres. Los temas en que las mujeres hicieron más hincapié fueron: condiciones laborales deficitarias en la cárcel, lugar central de lxs hijos e hijas en la vida de las mujeres no reconocido, mala atención de la salud, problemas en la visita (malos tratos, no respeto por parte de otras reclusas hacia los visitantes), así como desentendimientos y abandonos con lxs operadorxs judiciales, incluso lxs propixs abogadxs defensorxs.

Quedó evidenciado también a través de los tópicos diferenciales -al menos para nosotras las talleristasque el sistema penal y el sistema carcelario producen género y reproducen estereotipos hegemónicos sobre hombres y mujeres (Almeda y Bodelón, 2007). En este ámbito entonces, el dispositivo carcelario trabaja en forma muy precisa. La inmersión completa en este sistema provoca un reforzamiento de los estereotipos de lo que significa "ser mujer" y de lo que significa "ser hombre". Proceso que fue posible identificar en cómo durante las instancias de trabajo en los talleres los "derechos de las mujeres" son expresados y defendidos por las participantes, pero desde una perspectiva diferente a la nuestra, con énfasis distintos y visualizando causales de desventaja diferentes. Ser para los otros, por ejemplo, es profundamente reivindicado por estas mujeres. El argumento central expuesto como causal de la comisión de delitos es el bienestar de hijos e hijas. Sin embargo, esto no se hace en clave victimista, o invocando argumentos como el hambre, sino desde una posición que reivindica el "darles lo mejor", lo que incluye bienes de consumo incluso suntuarios. "Si el padre no se lo dá, se lo doy yo” (Fragmento de diario de campo, 22 de mayo de 2018).

Al mismo tiempo, la auto identificación como mujer, y como persona privada de libertad, no parecía poder unirse -al menos en los discursos presentes en la dinámica grupal- en una tercera categoría: la de mujeres privadas de libertad.

La caracterización de persona privada de libertad aparecía como neutra, tanto hombres como mujeres vivirían esa situación que se les impone, con todas las violencias, sufrimientos y carencias asociadas. 
Neutralidad que está asociada al androcentrismo del propio dispositivo como productor de subjetividad y que obtura la posibilidad de articular ambos conceptos. De tal manera, los procesos de alterización se radicalizan a través de la doxa que dictamina que la persona privada de libertad "correcta" -acertada - es varón.

Ellas, nuestras interlocutoras, participantes de los talleres, lo expresaron con claridad en diversas instancias. Manifestaron que los hombres privados de libertad "llevan mejor la cana" -aunque están en peores condiciones-, que son más limpios y prolijos que ellas, y que eso se puede constatar en el estado de las habitaciones donde se realizan las visitas íntimas en las diferentes cárceles. En palabras de una de ellas, con el asentimiento de las otras: "en las cárceles de varones están impecables, mientras que las de acá son una mugre" (Fragmento de diario de campo, 22 de mayo de 2018). Nuestras observaciones respecto de las condiciones materiales y de higiene en diversos recintos carcelarios no condicen con esta percepción manifiesta.

Estos planteos y la profundización en ellos para entenderlos durante las instancias de taller, provocaron en nosotras un dislocamiento al encontrarnos frente a lo inesperado a través de la experiencia. Porque si bien, desde las experiencias de investigación previas, coincidíamos con la afirmación de Beatriz Kalinsky (2009) que bien sintetiza de qué manera "La actividad criminal de una mujer es < anti-natural >, la desconoce en su propia identidad de mujer, y la lanza a un lugar de no-humanidad” (Kalinsky, 2009, s/p); aquí encontramos otro clivaje a tener en cuenta. Ya que, estar presa - "ser presa" - no las haría dejar de ser mujeres, pero se encuentran subsumidas a la categoría "varones privados de libertad", como elemento identitario que se presenta como más fuerte, no sólo en lo subjetivo, sino como grupo. Y es aquí donde el giro punitivo descrito anteriormente, con el etiquetamiento y las marcas que genera sobre los cuerpos de aquellas personas que son o han sido privadas de libertad, que son, utilizando las categorías nativas, nominados como: "chorros", "pichis", "ñeris", "perros" (todos términos masculinos o neutros), parece imponerse sobre la penetración del feminismo en este ámbito y en las subjetividades. Resulta así, oportuno sobre este aspecto, retomar una pregunta que se realiza la antropóloga ecuatoriana Coba “¿Cuál es la importancia del universo simbólico de lo masculino al interior de una prisión femenina?” (Coba, 2001, p. 5).

Otra situación en la que nos interesa detenernos y que se conecta con lo planteado más arriba sobre el lugar de lo trans, aconteció en el primer encuentro - que habíamos planificado fuera un espacio de presentación y generación de confianza fundamentalmente- Se trató de una instancia compleja, desordenada y en la que no pudimos llevar a cabo las dinámicas tal como lo teníamos planificado. Las mujeres fueron llegando en distintos momentos, el espacio que se nos cedió para trabajar no era el más adecuado, se dieron reencuentros y puestas al día (fundamentalmente, entre una de las talleristas y algunos participantes que ya conocía de trabajos anteriores en la institución); lo que generó que el encuadre fuera muy laxo y difícil de trazar.

El uso del masculino o "neutro" al decir de lxs participantes en el párrafo anterior no es casual, y se debe a que participó del primer taller un varón trans. Y esta participación generó una situación sobre el final de este primer encuentro que nos interesa desarrollar.

Rodrigo ${ }^{20}$ había tenido un vínculo muy cercano con una de las talleristas en épocas anteriores, durante el trabajo de campo para una investigación, y no se había dado luego otro contacto. Esta era la oportunidad para el reencuentro. Por otro lado, la operadora penitenciaria que realizaba la convocatoria entre las reclusas al taller, lo había invitado especialmente a participar y le había mencionado la presencia de esa "vieja conocida" en esa instancia. Este conocimiento previo fue expresado con claridad en esa instancia.

Él se sentó al lado mío y, ya finalizando el taller, se dio una conversación entre él y yo, en medio del desorden en el que todas hablaban entre ellas y con la otra tallerista.

Fue en ese momento en que Rosana, ${ }^{21}$ que yo conocía de antes (pero con la que no había tenido un vínculo de cercanía) estalló, generándose una situación muy violenta, en la que realizó una verdadera performance de su "ser carcelario", gesticulando y paseándose frente al resto de nosotras, gritando, arengando a las otras mujeres y colocando al varón trans en un lugar que podríamos calificar de total abyección en el sentido que le da Butler (Fragmento de diario de campo, 8 de mayo de 2018). 
En efecto, según Butler (2002, pp. 19-20):

Lo abyecto designa aquí precisamente aquellas zonas, " de la vida social que, sin embargo, están densamente poblada por quienes no gozan de la jerarquía de los sujetos, pero cuya condición de vivir bajo el signo de lo "invivible" es necesaria para circunscribir la esfera de los sujetos.

Según el diario de campo:

\begin{abstract}
En ese discurso a viva voz, expresó sus opiniones sobre la identidad de género, las prácticas sexuales y lo que ella consideraba la doble cara de este varón trans. Lo humilló entrando en detalles muy íntimos y lo calificó de estar representando un papel de víctima y de varón frente a nosotras, cuando en realidad no era tal. Él lloraba y se defendía como podía. Las otras mujeres cerraron filas tras la agresora, quien de a poco fue calmándose. Rodrigo se fue y nunca volvió a participar (Fragmento de diario de campo, 8 de mayo de 2018).
\end{abstract}

Esta situación fue disruptiva para quienes estábamos a cargo de moderar el taller. ${ }^{22}$ Nos tomó por sorpresa y generó un quiebre que desacomodó nuestra posición en el lugar. Pasamos a ser espectadoras de aquella escenificación de la violencia, sólo podíamos articular algunas palabras para intentar calmar la situación, que claramente no eran escuchadas, y la escena continuó hasta que Rosana se sintió apoyada por las otras y triunfadora. Luego de eso, bajó el tono y desarmó la performance corporal, dando algunas explicaciones, justificando su accionar y manifestando su cariño y conocimiento de Rodrigo, casi diciendo que lo hacía por su bien. La dinámica posterior hizo posible identificar, además de los aspectos de territorialidad, liderazgo y competencia -intrínsecos a la expulsión de una persona que no era reconocida por sus pares como participante en igualdad de condiciones de un taller solamente para mujeres - otros componentes ligados al despliegue de la sexualidad y el deseo hacia una otra proveniente del exterior.

Más allá de que la violencia entre pares es una práctica cotidiana en los centros de reclusión (Mesa Varela, 2019), esta manifestación específica nos resultó útil para pensar y fundamentalmente para poner en práctica la reflexividad.

Nos encontramos frente a una escenificación que nos golpeó con y en nuestras prenociones, en las que la -supuesta - unión en tanto mujeres buscando pelear por sus derechos, no significó la no violencia frente a lo otro, en este caso encarnada en Rodrigo. La coexistencia dentro del dispositivo carcelario, la propia situación de persona privada de libertad (que como veíamos más arriba, aparecía como ligadora a los hombres -biológicos- apresados y una reivindicación frente a la cada vez mayor violencia del Estado frente a quienes cometen delitos), no las unió a Rodrigo, sino que allí, la tecnología de género (De Lauretis, 2000) que dicotomiza y enuncia lo verdaderamente femenino y masculino, se expresó más potente frente a quien se ubica por fuera del binomio.

\title{
6. REFLEXIONES FINALES
}

Hasta aquí, presentamos dos de las actividades llevadas adelante por el equipo que permiten avizorar el entramado de alteridades insertas en la cárcel. Estas muestran parte del interjuego de relaciones de poder entre guardias policías, operadoras, operadores, presas, presos y familiares imbricadas en su dinámica.

En este sentido, el proceso de burocratización que parcela -en diversos roles con distinta dependencia y formación expresados en la brecha existente entre la norma y la práctica- el "tratamiento" dentro y en los márgenes de la cárcel, permite escindir el mandato correspondiente al funcionariado, en tanto cuerpo ejecutor de las obligaciones del Estado, de lo establecido por nuestro ordenamiento jurídico vigente.

Proceso que tiene condiciones de posibilidad en el contexto nacional de giro punitivo que se expresa en el acontecer al que nos referimos inicialmente -ejemplificado por la campaña "Vivir sin miedo"- y que en el contexto carcelario es encarnado por aquellas personas que allí trabajan. Quienes a la vez de ser asalariadxs precarixs resultan ejecutores directos de una política de Estado que no es enunciada como tal. 
Allí radica su fuerza, en la construcción de una realidad que al mismo tiempo que viola sistemáticamente los derechos humanos -amparándose en razones de seguridad y lógicas represivas- enuncia su defensa en diversas instancias internacionales. ${ }^{23}$ Enmascarando sus prácticas punitivas en una serie de medidas que podrían ser calificadas como "omisiones" al mismo tiempo que se deposita la responsabilidad en todas las personas imbuidas en la mecánica carcelaria.

En la situación de la revisoría que analizamos, es plausible identificar la reificación de la dicotomía hombre-mujer presentadas como categorías estables. Un claro ejemplo es cómo el cuerpo de la mujer trans es fragmentado, quedando la revisación de la genitalidad a cargo de un varón. Ese caso de discriminación extrema muestra un polo sobre cómo es percibido el cuerpo. Proceso que también se expresa a la interna del propio personal donde la masculinidad y el "ser policía" constituye la máxima jerarquía, siendo las operadoras -civiles y mujeres- las depositarias del mayor número de acciones de discriminación al momento de su ingreso a la función, reforzando y actualizando el androcentrismo del sistema.

Pero, como vimos en las interacciones presentadas, las representaciones del ser varón y el ser mujer -a partir de la encarnadura producto del dispositivo carcelario- precipitan también expresiones que dan cuenta de la extrema labilidad de estas categorías, que en su dislocación se contraponen a cualquier propuesta universalista e inhiben, en parte, las posibilidades de apropiación de los derechos de las mujeres privadas de libertad -tal como están definidos a través de las múltiples normas vigentes en el país - respecto al deber del Estado, en tanto garante del respeto de los derechos humanos de las personas bajo su tutela.

Las preguntas que obligan a detenerse en este asunto, surgen a partir del trabajo situado y podrían resumirse de la siguiente manera: ¿Qué nos une además de auto identificarnos como mujeres? ¿Qué nos separa además de que unas están privadas de la libertad y otras no? ¿En qué ámbitos resulta pertinente y estratégico trabajar sobre estas reflexiones? ¿Cómo capitalizar estos intercambios entre "ellas y nosotras" tanto desde el punto de vista teórico como identitario?

Al seguir a autoras como Segato (2013) y Azaola (2005), que aportan herramientas para el tratamiento de estos temas, a lo largo del texto proponemos mirar a las otras, mirarnos en las otras y mirarnos a nosotras. Y de estos ejercicios hermenéuticos es que nos hemos nutrido para pensar los encuentros y discusiones sobre y con las personas que forman parte del sistema penitenciario. En especial, con las otras mujeres, privadas de libertad.

Nos hemos encontrado con el desafío de comprender la situación actual de las mujeres presas en Uruguay, lo que no ha estado exento de dificultades. La necesidad de pelear contra sentidos hegemónicos atribuidos a qué puede ser considerado: delito, castigo y privación de libertad. Sentidos que tienen como característica constitutiva el androcentrismo y su correlato dicotómico en aproximarse a las mujeres como un todo heterogéneo, o al menos, como una categoría estable.

$\mathrm{Si}$ se observa la experiencia de trabajo con las internas de la Unidad Penitenciaria $\mathrm{N}^{\circ} 5$ vemos que nosotras, desde el equipo universitario, generamos una exclusión -al ser un taller (basado en una Guía) sobre y para "mujeres privadas de libertad"- dejando por fuera parte de la población que allí se encuentra recluida. Específicamente a los varones trans.

Esta situación resulta interesante para visibilizar y poner en cuestión los alcances de la categoría "mujer" y "mujer privada de libertad", cuando en términos identitarios no se aplica a todas las personas que se encuentran recluidas en una cárcel para mujeres. Observamos, en ese contexto situado, cómo los sistemas penal y carcelario -así como la vida delictiva- producen "una identidad" que aparece como núcleo cohesionador de pertenencia. Que como tal está constituida por símbolos y que es defendida y reivindicada a través del lenguaje, los comportamientos, las creencias, que se superpone a otras categorías como ser la de "mujeres", pero que no se mezcla con ella en aquello que es plausible de ser enunciado. 


\section{REFERENCIAS}

Almeda, E. y Bodelón, E. (Coords.) (2007). Mujeres y castigo. Un enfoque socio-jurídico y de género. Madrid: Dykinson.

Almeda, E. y Di Nella, D. (2017). Mujeres y cárceles en América Latina. Perspectivas críticas y feministas. Papers: revista de sociología, 102(2), 183-214. Recuperado de https://ddd.uab.cat/pub/papers/papers_a2017v102n2/p apers_a2017v102n2p183.pdf

Antony, C. (2000). Las Mujeres Confinadas. Estudio Criminológico sobre el rol genérico en la ejecución de la pena en Chile y América Latina. Santiago de Chile: Editorial Jurídica de Chile.

Antony, C. (2007). Mujeres invisibles: las cárceles femeninas en América Latina. Nueva sociedad, 208, 73-85. Recuperado de http://bdigital.binal.ac.pa/bdp/artpma/mujeres\%20delincuentes.pdf

Azaola, E. (2005). Las mujeres en el sistema de justicia penal y la antropología a la que adhiero. Cuadernos de antropologia social, 22, 11-25. Recuperado de http://www.pensamientopenal.com.ar/articulos/mujeressistemajusticia-penal-antropologia-que-adhiero

Bourdieu, P. (1967). Postfacio. En E. Panofsky, Architecture gothique et pensée scholastique (pp. 133-167). Paris: De Minuit.

Bourdieu, P. (1987). Los Tres Estados del Capital Cultural. Sociológica, 2(5), 11-17. Recuperado de https://sociolog iac.net/biblio/Bourdieu-LosTresEstadosdelCapitalCultural.pdf

Bourdieu, P. y Wacquant, L. (1992). Respuestas. Por una antropología reflexiva. México D.F.: Grijalbo.

Butler, J. (2002). Cuerpos que importan. Sobre los límites materiales y discursivos del sexo. Buenos Aires: Paidós.

Centro de Estudios Legales y Sociales (CELS), Ministerio Público de la Defensa de la Nación (MPDN), Procuración Penitenciaria de la Nación (PPN) (2011). Mujeres en prisión. Los alcances del castigo. Buenos Aires: Siglo XXI Editores.

CIDH (2009). Principios y buenas prácticas sobre la protección de las personas privadas de libertad en las Américas. Documento No 38. Recuperado de http://www.oas.org/es/cidh/mandato/Basicos/PrincipiosPPL.asp

$\mathrm{CIDH}$ (2017). Informe sobre medidas dirigidas a reducir el uso de la prisión preventiva en las Américas. Documento $\mathrm{N}^{\circ}$ 105. Recuperado de http://www.oas.org/es/cidh/informes/pdfs/PrisionPreventiva.pdf

CLADEM (2008). Violencia contra las mujeres privadas de libertad. Sistematización Regional. Argentina, Brasil, Chile, Colombia, Paraguay y Uruguay. Recuperado de http://americalatinagenera.org/newsite/images/cdr-document s/publicaciones/doc_747_documentodecladem.pdf

Coba, L. (2001). La muerte social: el Centro de Rehabilitación Social Femenino de Quito como situación humana. Quito: INREDH-CEE.

De Lauretis, T. (2000). Diferencias. Etapas de un camino a través del feminismo. Madrid: Horas y horas.

Fassin, D. (2018). Castigar. Buenos Aires: Adriana Hidalgo editora.

Folle, M. (2011). Situación de las mujeres privadas de libertad en el interior del pais Colonia, Durazno y Florida. Montevideo: Presidencia República Oriental del Uruguay-Naciones Unidas Uruguay. Recuperado de https://encuentrosenabril.files.wordpress.com/2016/04/folle-m-a-laino-n-montes-c-rodrc3adguez-m-2011 -situacic3b3n-de-las-mujeres-privadas-de-libertad-en-el-interior-del-pac3ads-colonia-durazno-y-florida.pdf

Folle, M. y Mesa Varela, S. (2016). Trazos de la Mesa de Trabajo sobre Mujeres Privadas de Libertad (2006-2014). En N. Montealegre Alegría (Coord.), El tiempo quieto. Mujeres privadas de libertad en Uruguay (pp. 127-143). Montevideo: JND, FHCE, CSIC, Udelar.

Folle, M. y Mosquera, S. (2016). Mujeres en pugna: prisión, dominación, resistencias. En N. Montealegre Alegría (Coord.), El tiempo quieto. Mujeres privadas de libertad en Uruguay (pp. 195-210). Montevideo: JND, FHCE, CSIC, Udelar.

Foucault, M. (2002). Vigilar y castigar: nacimiento de la prisión. Buenos Aires: Siglo XXI Editores.

Goffman, I. (2009). Internados. Ensayos sobre la situación social de los enfermos mentales. Buenos Aires: Amorrortu.

Guber, R. (2009). El salvaje metropolitano. Reconstrucción del conocimiento social en el trabajo de campo. Buenos Aires: Paidós. 
Guzmán, P. y Platero, R. (2012). Passing, enmascaramiento y estrategias identitarias: diversidades funcionales y sexualidades no normativas. En R. Platero (Ed.), Intersecciones: cuerpos y sexualidades en la encrucijada (pp. 125-158). Barcelona: Ediciones Bellaterra.

IMPO (2018). Fiscalia General de la Nación y nuevo Proceso Penal. Recuperado de https://www.impo.com.uy/fiscal ia-general-de-la-nacion-y-nuevo-proceso-penal/

Kalinsky, B. (2009). Antropología de la prisión preventiva. El caso del delito femenino. En Horizontes y convergencias. Lecturas históricas y antropológicas sobre derecho. Publicación de investigaciones cientificas de actualización continua. Buenos Aires: s. n. Recuperado de http://horizontesyc.com.ar/archivos/1251077413/antropologia_ de_la_prision_preventiva.pdf

Larrobla, F. y Mesa Varela, S. (2018). Efecto noviembre: discursos politicos y castigo a partir del nuevo Código Procesal Penal en Uruguay. En Primeras Jornadas "Estudios sobre el castigo", Bernal, Argentina.

Laurenzo, P. (s.d.). Violencia de género y derecho penal de excepción: entre el discurso de la resistencia y el victimismo punitivo. Recuperado de https://www.cijc.org/pt/seminarios/2011-Antigua/Documentos\%20CIJC/Libro\%20CGPJ\%20Patricia\% 20Laurenzo\%202007\%20-\%20VG\%20y\%20DPenal\%20de\%20excepci\%C3\%B3n\%20-\%20posici\%C3\%B3n \%20intermedia.pdf

Mesa Varela, S. (2012). La pasión, las heridas, las penas. Género, sexualidad y cuerpo en el Centro Metropolitano de Rehabilitación Femenino. (Tesis de grado), FHCE, UdelaR, Montevideo, Uruguay.

Mesa Varela, S. (2016). Mujeres privadas de libertad por tráfico y transportes de drogas en Uruguay: un análisis antropológico y de género. En N. Montealegre Alegría (Coord.), El tiempo quieto. Mujeres privadas de libertad en Uruguay (pp. 227-244). Montevideo: JND, FHCE, CSIC, Universidad de la República.

Mesa Varela, S. (2019). Bocas que (no) gritan: cuerpo y violencia en la cárcel de mujeres. Revista encuentros uruguayos, 11(2), 138-157. Recuperado de http://ojs.fhuce.edu.uy/index.php/encuru/article/view/143

Mesa de Trabajo sobre Mujeres Privadas de Libertad (2005). Mujeres privadas de libertad en el Uruguay. Informe sobre las condiciones de reclusión. Recuperado de http://www.inmujeres.gub.uy/innovaportal/file/19658/1/5_inform e_reclusas.pdf

Meza, F. (2016a). Guia Nuestros Derechos. Montevideo: CSEAM, Universidad de la República.

Meza, F. (2016b). Pertinencia de escribir una guía con perspectiva de género para las mujeres privadas de libertad sobre sus derechos humanos. En N. Montealegre Alegría (Coord.), El tiempo quieto. Mujeres privadas de libertad en Uruguay (pp. 145-163). Montevideo: JND, FHCE, CSIC, Universidad de la República.

Meza, F. (2018). Guia Nuestros Derechos. Montevideo: CSEAM, Universidad de la República.

Montealegre Alegría, N. (Coord.) (2016a). El tiempo quieto. Mujeres privadas de libertad en Uruguay. Montevideo: JND, FHCE, CSIC, Universidad de la República. Recuperado de http://www.fhuce.edu.uy/images/genero_cu erpo_sexualidad/Publicaciones/FHCE_MPL_2016-11-14-LR_web.pdf

Montealegre Alegría, N. (2016b). La visita carcelaria: género, pichis y ritos de paso en el Uruguay. En N. Montealegre Alegría (Coord.). El tiempo quieto. Mujeres privadas de libertad en Uruguay (pp. 177-194). Montevideo: JND, FHCE, CSIC, Universidad de la República.

Montealegre Alegría, N. (2019). De la cárcel y las mujeres... Hemisferio izquierdo, Rebelión feminista, 28. Recuperado de https://www.hemisferioizquierdo.uy/single-post/2019/03/11/De-la-c\%C3\%A1rcel-y-las-mujeres\%E2\%80 $\%$ A6

Naciones Unidas, CEDAW (2016). Observaciones finales sobre los informes periódicos octavo y noveno combinados del Uruguay. Recuperado de http://acnudh.org/comite-para-la-eliminacion-de-la-discriminacion-contra-la-mujercedaw-uruguay-2016/

Naciones Unidas (2018). Recopilación sobre el Uruguay. Informe de la Oficina del Alto Comisionado de las Naciones Unidas para los Derechos Humanos. Recuperado de https://undocs.org/pdf?symbol=es/A/HRC/WG.6/32/U $\mathrm{RY} / 2$

Nikken, P. (s/f). El concepto de derechos humanos. Recuperado de http://www.derechoshumanos.unlp.edu.ar/assets/f iles/documentos/el-concepto-de-derechos-humanos.pdf 
Nogueira, H. (2003). Los derechos esenciales o humanos contenidos en los tratados internacionales y su ubicación en el ordenamiento jurídico nacional: doctrina y jurisprudencia. Revista Ius et praxis, 9(1). Recuperado de https:/ /www.corteidh.or.cr/tablas/r10635.pdf

Nowack, M. (2005). Derechos Humanos: manual para parlamentarios. Ginebra: Unión Inter-Parlamentaria y Oficina del Alto Comisionado para los Derechos Humanos de las Naciones Unidas. Recuperado de http://archive.hrea.org/erc/Library/display_doc.php?url=http\%3A\%2F\%2Fwww.ipu.org\%2FPDF\%2Fpu blications\%2Fhr_guide_sp.pdf\&external=N

Preciado, B. (2009). Basura y género. Mear/Cagar. Masculino/Femenino. Parole de Queer, 2, 14-17.

Scarfo, F. (2003). El Derecho a la educación en las cárceles como garantía de una Educación en Derechos Humanos. Revista del Instituto Interamericano de Derechos Humanos, 36, 291-324. Recuperado de http://www.corteidh.o r.cr/tablas/r06835-11.pdf

Segato, R. (2013) La crítica de la colonialidad en ocho ensayos y una antropología por demanda. Buenos Aires: Prometeo Libros.

Tait, S. (2011). A typology of prison officer approaches to care. European journal of criminology, 6(8), 440-454.

UNODC (2015). Reglas Minimas de las Naciones Unidas para el tratamiento de los Reclusos (Reglas Nelson Mandela). Recuperado de https://www.unodc.org/documents/justice-and-prison-reform/Nelson_Mandela_Rules-S-ebo ok.pdf

Uruguay. Comisionado Parlamentario Penitenciario. (2018). Informe anual. Recuperado de https://parlamento.gub. uy/sites/default/files/DocumentosCPP/ARMADO\%20interior\%20inf\%202017\%20FINAL\%20WEB.pdf

Uruguay. Ministerio del Interior (2011). Informe sobre buenas prácticas en materia penitenciaria en la República Oriental del Uruguay. Recuperado de https://medios.presidencia.gub.uy/jm_portal/2011/noticias/NO_A828 /informecarceles.pdf

Vigna, A. (2008). Género y delito. Reflexiones en torno a la criminalidad femenina en Uruguay. Tesis de grado. FCS, UdelaR, Montevideo.

Vigna, A. (2011a). Discriminación hacia personas privadas de libertad y liberadas. En Hacia un Plan Nacional contra el Racismo y la Discriminación. MEC-. AECID/PNUD. Recuperado de http://www.inae.gub.uy/innovaportal /file/10904/1/informe_privados_de_libertad.pdf

Vigna, A. (2011b). Persistencia y abandono del mundo del delito: diferencias de género en los procesos de desistimiento. Tesis de Maestría en Sociología. FCS, UdelaR, Montevideo, Uruguay.

Vigna, A. (2012). Análisis de datos del I Censo Nacional de Reclusos, desde unaperspectiva de Género y Derechos Humanos. Montevideo: INMUJERES. Recuperado de http://www.inmujeres.gub.uy/innovaportal/file/24956/1/infocen so.pdf

Wacquant, L. (2010). Castigar a los pobres. El gobierno neoliberal de la inseguridad social. Barcelona: Gedisa.

Wacquant, L. (2010b). Las cárceles de la miseria. Buenos Aires: Manantial.

República Oriental del Uruguay. Presidencia (2018). Discurso en la ONU. Vázquez llamó a los líderes más poderosos del mundo a combatir el cambio climático y a cumplir el Acuerdo de París. Recuperado de http://presidencia.gub.uy/ comunicacion/comunicacionnoticias/vazquez-discurso-onu-cambio-climatico-acuerdo-paris

\section{Notas}

1 A lo largo del artículo, utilizaremos esta categoría, así como las categorías nativas (tanto institucionales, como de las "internas" y las nuestras), para evidenciar la diversidad de apelativos correspondientes a este grupo de personas, de acuerdo a los colectivos imbricados en la dinámica carcelaria y las reflexiones asociadas.

2 Equipo Género, Memoria y Derechos Humanos, coordinado por la Prof. Graciela Sapriza. El artículo es parte de los resultados de los proyectos de la Universidad de la República: "Cárcel: el paradigma de la rehabilitación revisitado", Proyectos de Extensión Relativos a Derechos Humanos 2017-2018 (CSEAM) y "Lo carcelario en Uruguay. Una mirada crítica a los procesos de cambio desde una perspectiva de Género y DDHH (2005-2017)”, Espacio Interdisciplinario. 
3 Se tiene en cuenta lo planteado por Foucault: "Hay que recordar también que el movimiento para reformar las prisiones, para controlar su funcionamiento, no es un fenómeno tardío. No parece siquiera haber nacido de una comprobación de fracaso debidamente establecido. La "reforma" de la prisión es casi contemporánea de la prisión misma. Es como su programa. La prisión se ha encontrado desde el comienzo inserta en una serie de mecanismos de acompañamiento, que deben en apariencia corregirla, pero que parecen formar parte de su funcionamiento mismo" (Foucault, 2002, p. 236).

4 Los gobiernos y otros titulares de deberes tienen la obligación de respetar, proteger y satisfacer los derechos humanos, que constituyen la base legal para la reivindicación de derechos y la demanda de reparación en caso de incumplimiento (Nowack, 2005, p. 1).

5 Uruguay viene recibiendo recomendaciones del Comité contra la Tortura, del Comité de Derechos Humanos y del Comité para eliminar la Discriminación contra la mujer respecto a la situación de las personas privadas de libertad y de las mujeres reclusas. El equipo de las Naciones Unidas en el país dijo que el Uruguay enfrentaba una crisis del sistema penitenciario y que la cantidad de personas privadas de libertad había aumentado de manera sostenida. También dijo que aún persistían centros con hacinamiento, encierro prolongado y confinamiento en solitario, y preocupaban los episodios violentos dentro de las cárceles, debido a las malas condiciones de reclusión (Naciones Unidas, 2018, p. 18). Cabe resaltar la preocupación del Comité para eliminar la discriminación contra la mujer sobre las mujeres reclusas en Uruguay. El Comité le recuerda las Reglas de las Naciones Unidas para el tratamiento de las Reclusas y Medidas No Privativas de la Libertad para las Mujeres Delincuentes (Reglas de Bangkok) y le recomienda que refuerce la vigilancia de las mujeres en los centros de detención a fin de evitar las violaciones de los derechos humanos y garantice la disponibilidad de servicios de asesoramiento jurídico y vías de recurso eficaces (Naciones Unidas, 2016, p. 43-44).

6 "La población penitenciaria no ha parado de aumentar desde 1999, momento en que el país tenía unos 4.000 presos, mientras en diciembre de 2017 teníamos 10.241" (Comisionado Parlamentario Penitenciario, 2018, p.28).

7 La Comisión Interamericana de Derechos Humanos recuerda en su Informe sobre medidas dirigidas a reducir el uso de la prisión preventiva en las Américas de 2017 "que la prisión preventiva debe partir de la consideración al derecho a la presunción de inocencia y tener en cuenta la naturaleza excepcional de esta medida; además, debe aplicarse de conformidad con los criterios de legalidad, necesidad y proporcionalidad. La privación de libertad de la persona imputada debe tener un carácter procesal, y, en consecuencia, sólo puede fundamentarse en sus fines legítimos, a saber: asegurar que el acusado no impedirá el desarrollo del procedimiento ni eludirá la acción de la justicia” (CIDH, 2017, p. 15).

8 Actualmente está en proceso de discusión un proyecto de ley sobre el Sistema Penitenciario que establecería el pasaje del INR a la órbita del Ministerio de Educación y Cultura. El proyecto ha recibido amplias críticas de diversas organizaciones y del propio Comisionado parlamentario. Entre otros aspectos se destaca la falta de armonización con el derecho laboral del país, la referencia a las mujeres únicamente en relación a su maternidad y la reducción -sin ningún tipo de evaluación ni consideración- del tiempo de posible estadía con sus hijos a los 24 meses y que entre otros problemas no se ajusta en sus términos al derecho internacional suscrito por el país (Montealegre Alegría, 2019).

9 Ámbito de trabajo interinstitucional con la participación de organismos gubernamentales y organizaciones de la sociedad civil, entre otros. La primera constitución estuvo integrada por: Ministerio del Interior, Dirección Nacional de Cárceles, Patronato Nacional de Encarcelados y Liberados, Patronato Departamental de Canelones, Comisionado Parlamentario para el Sistema Carcelario, Programa Salud de la Mujer y Género del Ministerio de Salud Pública, Comisión Especial de Equidad y Género de la Cámara de Representantes, Consejo de Educación Secundaria, SERPAJ, Instituto Nacional de las Mujeres, ANIMA, CLADEM (Comité de América Latina y Caribe para Defensa de los Derechos Humanos de las Mujeres), Junta Nacional de Drogas - Secretaría Nacional de Drogas. La constitución interna ha ido variando a lo largo de los años, incorporando organizaciones diversas.

10 Desde otra perspectiva en el ámbito penal, este proceso se ha presentado como "el punitivismo vindicativo", tal como plantea la Catedrática de Derecho Penal de la Universidad de Málaga, Dra. Patricia Laurenzo (Laurenzo, s/d).

11 Partido tradicional con alianzas de larga data con los sectores económicos vinculados a la producción ganadera y agropecuaria del país, opositor a la llamada "agenda de derechos" en base a un discurso conservador y de derecha que en los últimos años ha permitido una alianza a su interna con sectores evangélicos y contrarios al derecho a decidir de las mujeres sobre su cuerpo con énfasis en la oposición radical a la interrupción voluntaria del embarazo.

12 Tomando el concepto de Foucault (2002) y el trabajo reciente de Fassin sobre el mismo.

13 La masividad de las manifestaciones por el 8 de marzo y el ni una menos son algunos ejemplos de la masividad del movimiento feminista en el presente en el país. A estas expresiones se suman las múltiples organizaciones feministas, de mujeres y LGTTBI existentes en el país y algunos de los logros obtenidos, como por ejemplo la ley trans. Todos elementos en disputa por parte de sectores conservadores en consonancia con el acontecer en la región.

14 El hecho de que las mujeres en América Latina sean quienes más visitan y las menos visitadas ha sido objeto de reflexión de las académicas feministas en diversos contextos. A modo de ejemplo se puede consultar el trabajo de Carmen Antony (2007).

15 Como por ejemplo el Caso Cantoral Benavides vs Perú; Caso Gómez Paquiyauri vs Perú; Caso Instituto de reeducación del menor vs Paraguay, entre otros. 
16 Pichi es el vocablo despectivo que condensa el estigma de persona pobre y delincuente. Sobre esta denominación y su uso en contextos carcelarios, ver: Montealegre Alegría (2016b, p. 183).

17 El diseño de la Guía de Derechos para Mujeres Privadas de Libertad (CSEAM-Universidad de la República) estuvo a cargo de Fabiana Larrobla.

18 La Guía pudo estar en condiciones de ser distribuida en las unidades penitenciarias a partir del consenso con el Ministerio del Interior sobre sus contenidos. Sin embargo, algunos de estos contenidos referidos al derecho de recibir refuerzo en la alimentación de mujeres embarazadas, portadoras de VIH y aquellas con niños a su cargo dentro de la cárcel, fueron censurados posteriormente sin notificarnos.

19 Entendida como la reflexión crítica de la investigadora "acerca de sus supuestos, su sentido común, su lugar en el campo y las condiciones históricas y socioculturales bajo las que lleva a cabo su labor” (Guber, 2009, p. 177).

20 A los efectos de este apartado fueron cambiados los nombres propios.

21 El nombre utilizado a los efectos de este texto fue cambiado. Con ese apelativo se identifica a una de las mujeres participantes del taller con marcado liderazgo y que es identificada como una "brazo gordo", es decir: una mujer fuerte, con poder dentro de su sector y considerada líder por gran parte de las otras internas.

22 Docentes: Gabriela Fernández y Serrana Mesa Varela.

23 En su discurso ante la Asamblea General de las Naciones Unidas, el 26 de septiembre de 2018, el Presidente Vázquez reafirmó el compromiso de Uruguay con un sistema internacional más participativo, justo, equilibrado y multipolar. También destacó el irrenunciable compromiso de Uruguay con la promoción y protección de los derechos humanos, entre otros (República Oriental del Uruguay. Presidencia, 2018). 